\title{
Applicability of Macroscopic Wear and Friction Laws on the Atomic Length Scale
}

\author{
S. J. Eder, ${ }^{1, *}$ G. Feldbauer, ${ }^{1,2}$ D. Bianchi, ${ }^{1}$ U. Cihak-Bayr, ${ }^{1}$ G. Betz, ${ }^{2}$ and A. Vernes ${ }^{1,2}$ \\ ${ }^{1}$ Austrian Center of Competence for Tribology, AC2T research GmbH, Viktor-Kaplan-Straße 2, 2700 Wiener Neustadt, Austria \\ ${ }^{2}$ Institute of Applied Physics, Vienna University of Technology, Wiedner Hauptstraße 8-10/134, 1040 Vienna, Austria
}

(Received 28 November 2014; revised manuscript received 13 February 2015; published 8 July 2015)

\begin{abstract}
Using molecular dynamics, we simulate the abrasion process of an atomically rough $\mathrm{Fe}$ surface with multiple hard abrasive particles. By quantifying the nanoscopic wear depth in a time-resolved fashion, we show that Barwell's macroscopic wear law can be applied at the atomic scale. We find that in this multiasperity contact system, the Bowden-Tabor term, which describes the friction force as a function of the real nanoscopic contact area, can predict the kinetic friction even when wear is involved. From this the Derjaguin-Amontons-Coulomb friction law can be recovered, since we observe a linear dependence of the contact area on the applied load in accordance with Greenwood-Williamson contact mechanics.
\end{abstract}

DOI: 10.1103/PhysRevLett.115.025502

PACS numbers: 62.20.Qp, 31.15.xv, 68.35.Af, 81.40.Pq

On the macroscale a manifold of wear models and equations exist [1]. Barwell gave a set of three different empirical wear laws describing the wear-in, the steadystate, and the wear-out regimes, respectively [2]. Most commonly, wear laws are phenomenological relationships based on contact-mechanical considerations. Several of these steady-state wear models were developed around the middle of the 20th century and predict a linear dependence of the wear volume on the load and the sliding distance [3-6]. They all typically depend on at least one empirical parameter (e.g., the wear coefficient) that cannot actually be derived, so their predictive power is severely limited.

Over the last decades, effects at the nanoscale have received increasing attention [7]. Various experiments, which typically investigate single-asperity contacts established in an atomic force microscope (AFM), have shown that established macroscopic wear laws are not readily applicable at the atomic length scale [8-10]. Currently, the most effort in nanotribology is directed toward singleasperity contacts due to their fundamental importance and the possibility of well-defined experiments with AFMs. However, considering applications in nanotechnology such as nano- or microelectromechanical systems [11], many open questions remain such as the influence of the multiasperity contact area on the acting wear mechanisms.

The Amontons-Coulomb law [12] states that, for moderate sliding velocities, the friction force is linearly dependent on the applied load via the coefficient of friction $(\mathrm{CoF})$ and independent of the apparent contact area. By recognizing the importance of roughness in contacts, Bowden and Tabor noted that the friction force is proportional to the contact area of the involved asperities via the

Published by the American Physical Society under the terms of the Creative Commons Attribution 3.0 License. Further distribution of this work must maintain attribution to the author(s) and the published article's title, journal citation, and DOI. effective shear strength [13]. The applicability of such macroscopic friction laws to nanoscale contacts must be subject to close scrutiny since understanding the relevant nanoscopic friction phenomena remains a challenge $[14,15]$.

In this work, we apply a recently proposed modeling and evaluation approach based on molecular dynamics (MD) simulations $[16,17]$ to quantify the wear depth, the contact area, and the friction force that arise during the two-body wear of a nanorough $\mathrm{Fe}$ surface due to multiple hard abrasive particles. The discussion of the wear and friction laws that are applicable to dry multiasperity abrasion at the nanoscale is the purpose of this Letter.

All simulations were performed using the open-source MD code LAMMPS [18] and a Finnis-Sinclair potential [19] for the bcc Fe substrate interactions. Figure 1(a) shows a snapshot of the surface with lateral dimensions of $28.5 \times$ $28.5 \mathrm{~nm}^{2}$ (replicated periodically) and Gaussian roughness with a root-mean-squared height of $0.8 \mathrm{~nm}$ before the abrasion process starts. The randomly oriented rigid cubic abrasive particles with an edge length of $4.2 \mathrm{~nm}$ are then dragged across the surface at a velocity of $v^{(\max )}=80 \mathrm{~m} / \mathrm{s}$ for $15 \mathrm{~ns}$ and an angle of $\approx 7^{\circ}$ with the box edge so that the particles do not immediately rework their own wear tracks. Their relative positions are fixed and particle rotation is not allowed, which corresponds to two-body wear or grinding with hard, bound abrasives. This process is simulated at constant loads of 81.5, 244.5, 407.5, 611.25, and $815 \mathrm{nN}$, equivalent to normal pressures of $0.1-1 \mathrm{GPa}$ obtained via a division by the nominal contact area, similar to Refs. [20,21]. Because of their rigidity, the abrasive particles themselves are not subject to wear. The interaction between the abrasives and the $\mathrm{Fe}$ surface was modeled using a Lennard-Jones potential with $\varepsilon=0.125 \mathrm{eV}$ and $\sigma=0.2203 \mathrm{~nm}$, leading to weak adhesion. Equivalent simulations were carried out using abrasives of spherical shape with a radius of $3.25 \mathrm{~nm}$. The base of the substrate is 
thermostated to $300 \mathrm{~K}$ using a Langevin thermostat, but during the simulations the average temperature of the substrate lies between 340 and $500 \mathrm{~K}$, depending on abrasive particle geometry and load. The MD-typical disregard of some electronic effects, in particular their contribution to the material's thermal conductivity, results in these high temperature gradients within the substrate, which in turn may lead to somewhat overestimated surface temperatures and wear rates.

During the simulations, any atom with a momentary advection velocity of $v<0.1 v^{(\max )}$ is labeled as part of the substrate, with $0.1 v^{(\max )} \leq v \leq 0.9 v^{(\max )}$ as part of the shear zone, and with $v>0.9 v^{(\max )}$ as part of a wear particle, see Fig. 1(b). This approach allows the dynamic partition of the nonrigid system components into deformation zones with different crystallographic features, as justified in Ref. [16]. The number of atoms in the wear particles, multiplied with a per-atom volume derived from a Fe bcc unit cell and divided by the lateral cross section of the simulation box, yield the mean wear depth $h_{w}$. We found that the time development of $h_{w}(t)$ can be best

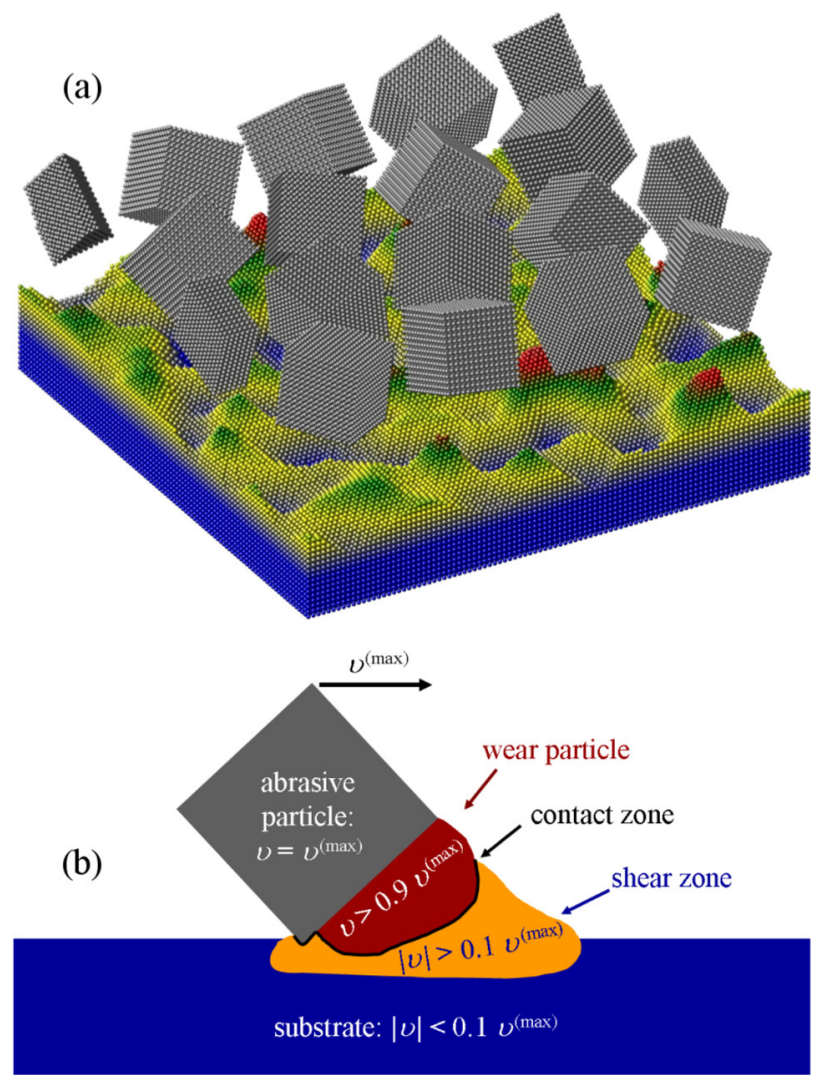

FIG. 1 (color online). (a) Initial system configuration with cubic abrasive particles. The abrasives are shown in gray, and the Fe substrate is colored according to the local height, where blue denotes low and red denotes high. (b) Overview of the deformation zones to which an atom can be attributed according to its momentary advection velocity $v$, where $v^{(\max )}$ is the sliding velocity of the abrasive particles. expressed as a combination of Barwell's wear-in and steady-state contributions

$$
h_{w}(t)=w_{0} \tau_{w}\left[1-\exp \left(-\frac{t}{\tau_{w}}\right)\right]+w_{c} t
$$

which reproduces our data more closely than, e.g., a squareroot time dependence found valid in Ref. [22]. In Eq. (1), $w_{0}$ is the initial wear (depth) rate at $t=0$ that dominates the run-in period characterized by the time constant $\tau_{w}$, and $w_{c}$ is the steady-state wear (depth) rate. The separate use of the two terms in Eq. (1) has led to a feasible experimental methodology to determine macroscopic wear coefficients from measured data [23], while a combination of both terms has been used to predict the experimental run-in and stationary wear of metals in ball-on-disk test configurations $[24,25]$. According to our best knowledge, we can show here for the first time that this combination of macroscopic wear laws also holds on the nanoscopic length scale, as can be seen in Fig. 2.

Most of the height reduction by wear takes place during the first nanosecond in all cases. With spherical abrasives and low loads, hardly any wear takes place, so the respective final topography is almost entirely a product of an atom rearrangement from asperity tips to pits. The time constant $\tau_{w}$ rises linearly with load when using spherical abrasives, ranging from 0.02 to $0.24 \mathrm{~ns}$, while for cubic abrasives it is practically load independent at $\tau_{w} \approx 0.22 \mathrm{~ns}$. The initial wear rates $w_{0}$ lie in the range of $0.1-1.3 \mathrm{~m} / \mathrm{s}$, most likely influenced by the steep asperity flanks of the initial substrate topography. Here, we observe

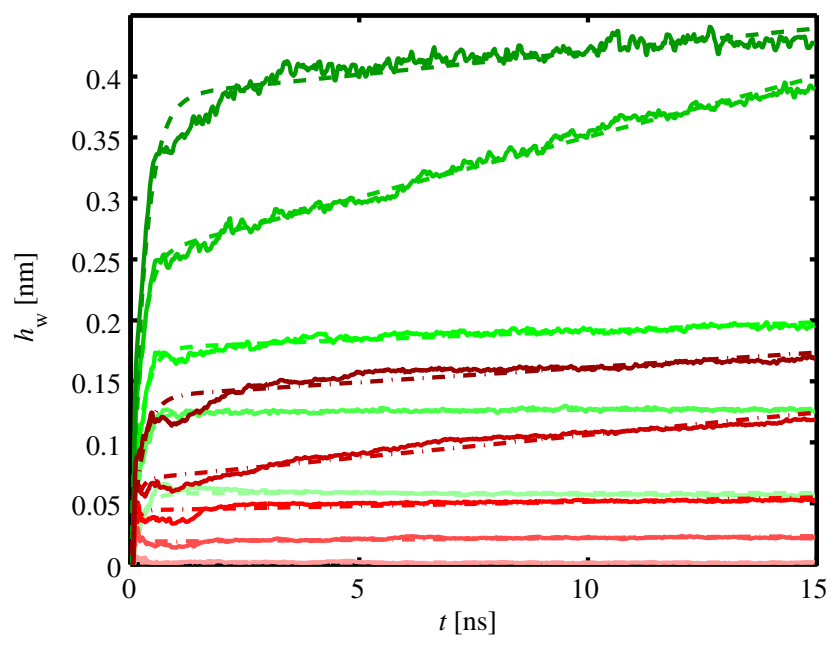

FIG. 2 (color online). Time dependence of the wear depth $h_{w}$ calculated from simulation data (solid curves) fitted to the combined Barwell wear law in Eq. (1) for several applied loads. Green curves with dashed fits denote cubic abrasives; red ones with dot-dashed fits denote spherical abrasives. Light hues represent low loads; dark ones represent high loads. 
a sublinear load dependence that is more pronounced for the spherical geometry than for the cubic abrasives. Since $w_{0}$ dominates the wear behavior for only approximately $1 \mathrm{~ns}$ in our simulations, it cannot be compared to wear rates measured during (much longer) experimental run-in periods. We therefore focus on validating the results for the steady-state wear rates $w_{c}$, which, at $0.1-4 \mathrm{~mm} / \mathrm{s}$, are approximately 3 orders of magnitude smaller than the initial wear rates $w_{0}$ and rise slightly superlinearly with load, which might be explained with thermal activation [26]. Although the Finnis-Sinclair potential used for the Fe interactions could reproduce the corresponding processes, our mean surface temperatures of up to $700 \mathrm{~K}$ may not be sufficient for overcoming the activation energies taken from Ref. [26].

We compare our fitted wear rates with two different experimental works where sufficient data and information are available. A ten-year experimental study on the wear of soft gliding metals against hard gun steel at high sliding velocities using a sophisticated high speed pin-on-disk test device led to a vast amount of wear data [27,28]. A conversion of our simulated steady-state wear rates to the units used in Ref. [28] yields values of $0.004-0.16 \mathrm{~mm}^{3} / \mathrm{m}$ depending on load, which must be compared to experimental rates of $0.002-1.5 \mathrm{~mm}^{3} / \mathrm{m}$ obtained for a similar sliding velocity, but with the pressure range being an order of magnitude smaller than ours. In an altogether different example involving high pressure grinding rolls used in the mineral processing industry [29], wear rates of $0.08-0.64 \mathrm{~mm}^{3} / \mathrm{m}$ were reported for pressures up to $1 / 100$ th of our maximum value. By estimating the lateral size of the effective contact zone between the rollers $\left(\approx 30-150 \mathrm{~mm}^{2}\right)$, the maximum range of our corresponding simulated data converts to volumetric wear rates of $0.04-7.5 \mathrm{~mm}^{3} / \mathrm{m}$. Corrected for the applied load, our data fall into the lower ends of both experimental wear rate ranges as expected, since both examples exhibit high wear rates, while typical grinding processes will lead to markedly lower ones. We thus conclude that the results of our calculations lie well within the bandwidth of experimental macroscopic wear measurements.

In previous work, the authors have shown that the friction force can be expressed as a superposition of the load-dependent Amontons-Coulomb law, the contact-areadependent Bowden-Tabor term, and the load-independent Derjaguin offset [30]. The resulting three-term kinetic friction law for nano-tribological systems

$$
F(L)=F_{0}+\tau A_{c}(L)+\mu L
$$

describes mixed and boundary lubrication as well as the transition between these lubrication regimes via three independent constitutive system parameters: the Derjaguin offset $F_{0}$, the effective shear strength $\tau$, and the $\mathrm{CoF} \mu$. For this, classical MD was combined with the smooth particle method (SPM) to estimate the load-dependent real contact area $A_{c}(L)$ [31]. In the case of boundary lubrication where no solid-solid contact occurs, it was demonstrated that the three-term kinetic friction law turns into the Derjaguin form [32,33] $F(L)=F_{0}+\mu L$; i.e., a nonvanishing Derjaguin offset $F_{0}$ complements the Amontons-Coulomb term $\mu L$, where $L$ is the applied load. Furthermore, we found that $F_{0}$, commonly attributed to the adhesion in the presence of a lubricant at $L=0$, correlates with the disorder in the lubricant [32] and is exponentially dependent on the normalized sliding resistance area in boundary-lubricated systems [33].

In our present simulations there always exists a nonvanishing contact area between the atomically rough surface and the abrasive particles independently of the applied load, see Fig. 3. In contrast to the combined MD and SPM scheme [31], here we first determine the number of atoms defining the contact zone, see Fig. 1(b) and Ref. [16] for details. The load-dependent multiasperity contact area $A_{c}(L) \neq 0(\forall L \geq 0)$ is then calculated by multiplying this number with the estimated average area of $8.15 \AA^{2}$ assigned to a single Fe atom on the bcc (001) surface, a procedure similar to that applied in Refs. [14,34]. More accurate computational schemes, such as a Voronoi tessellation [32] or an ab initio approach [35], lead to only slightly different contact areas.

Inspecting the dependence of the friction force $F(L)$ on the resulting multiasperity contact area $A_{c}(L)$ in Fig. 3(a), one immediately observes that $F(L)$ is essentially directly proportional to $A_{c}(L)$, although the vanishing of both quantities would require the load to become negative. The points representing $A_{c}(0)$ are indicated by arrows in Fig. 3(a) and can also easily be deduced from Fig. 3(b).
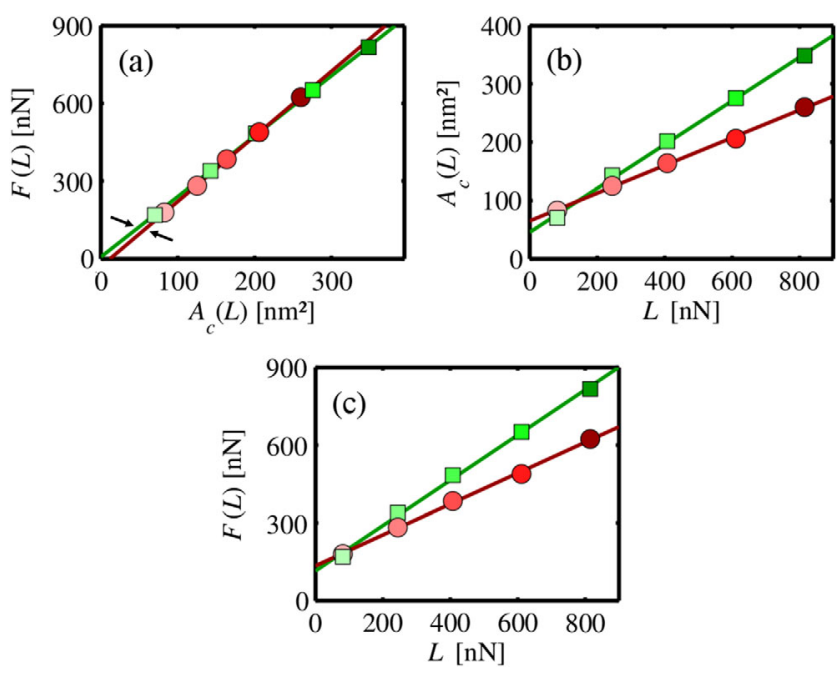

FIG. 3 (color online). (a) Friction force versus contact area. (b) Contact area versus load. (c) Friction force versus load. Green squares and red circles denote cubic and spherical abrasives, respectively. Lines are linear fits to the data, and arrows indicate the contact area at zero load. 
In accordance with the three-term kinetic friction law, this means that the Bowden-Tabor term alone

$$
F(L)=\tau A_{c}(L)
$$

can describe the kinetic friction in the dry nanoscopic abrasion process. Note that $F_{0}$ becomes zero as $F(L)=0$ for $A_{c}(L)=0$. The slope of the linear $F(L)$-versus- $A_{c}(L)$ relationship corresponds to the effective shear strength $\tau$, a load-independent constitutive system parameter that, as shown in Table I, is slightly dependent on the shape of the abrasive particles. The values of $\tau$ are higher than the shear strength of Fe. The reason for this is that the effective shear strength does not merely describe the shearing of $\mathrm{Fe}$ nanoasperities in the sense of cleaving, but rather the kinetic friction of the entire nanotribological system, which also includes the rigid abrasives, the formation of a dynamic shear zone, as well as the energy-consuming separation and redeposition of small clusters of atoms.

The pioneering single-asperity nanotribological studies performed in the 1990s using either a surface forces apparatus [36] or an AFM [37] have led the way to the successful application and validation of Eq. (3) at nanoscopic length scales $[38,39]$. Here, it seems that the singleasperity contributions to the friction force sum up into a single Bowden-Tabor term, as was already predicted in the late 1930s [40], and accordingly Eq. (3) remains valid at the nanoscale for dry multiasperity contacts. In contrast to other MD work dealing with single- and multiasperity contacts [14,41,42], we also quantify wear in our simulations.

The multiasperity contact area $A_{c}(L)$ is found to vary linearly with the applied load $L$, i.e.,

$$
A_{c}(L)=A_{c}(0)+a_{c} L,
$$

as depicted in Fig. 3(b), where $a_{c}$ is the load independent slope of the $A_{c}(L)$-versus- $L$ relationship, and $A_{c}(0)>0$ is the multiasperity contact area at zero load. As can be seen in Table I, both quantities $a_{c}$ and $A_{c}(0)$ depend on the geometry of the abrasive particles. The linear dependence of the real nanoscopic contact area on the applied load $L$ was also found in the wearless multiasperity MD simulations performed in Refs. [14,42], but it has not been observed in single-asperity experimental nanotribology [39]. At the macroscopic length scale, Eq. (4) was first experimentally found by Bowden and Tabor [40], and its validity was theoretically proven by

TABLE I. Best-fit parameters for Eqs. (3), (4), and (5).

\begin{tabular}{lccccc}
\hline \hline Abrasive & $\tau(\mathrm{GPa})$ & $A_{c}(0)\left(\mathrm{nm}^{2}\right)$ & $a_{c}\left(\mathrm{~nm}^{2} / \mathrm{nN}\right)$ & $F_{0}^{*}(\mathrm{nN})$ & $\mu^{*}$ \\
\hline Spherical & 2.51 & 65.1 & 0.237 & 133.6 & 0.596 \\
Cubic & 2.33 & 45.7 & 0.375 & 113.9 & 0.874 \\
\hline \hline
\end{tabular}

Greenwood and Williamson [43] assuming normally distributed heights of the Hertzian asperities on the rough surfaces in dry contact. The reason for the intersection between the lines in Fig. 3(b) can be explained as follows. At high loads, the sharper cubic abrasives can penetrate deeper into the substrate, while also producing larger wear particles that contribute to the total contact area. At low loads, the higher conformity of the contact between the spherical abrasives and the surface leads to a larger contact area at zero load than for the point or edge contacts of the cubic abrasives.

Substituting Eq. (4) into Eq. (3), the Derjaguin generalization of the Amontons-Coulomb friction law is formally recovered as

$$
F(L)=\tau A_{c}(0)+\left(\tau a_{c}\right) L \equiv F_{0}^{*}+\mu^{*} L .
$$

In contrast to the Derjaguin offset $F_{0}$ and the $\operatorname{CoF} \mu$ in the Derjaguin friction law, none of the quantities $F_{0}^{*} \equiv \tau A_{c}(0)$ and $\mu^{*} \equiv \tau a_{c}$ introduced here can be considered constitutive system parameters, although they can even survive in dry contact situations [44] and are independent of the load, because they depend on each other, namely, $F_{0}^{*} / \mu^{*}=A_{c}(0) / a_{c}$. Note that when comparing the independently fitted sets of parameters in Table I, the equivalence $\mu^{*}=\tau a_{c}$ is reproduced, while the values on the left and right side of $F_{0}^{*}=\tau A_{c}(0)$ differ by $5 \%-20 \%$ since $F_{0}^{*}$ is an extrapolation value. When plotting only the frictionversus-load behavior without any knowledge of the real contact area, as in Fig. 3(c), one might erroneously conclude that the Derjaguin friction law sufficiently describes our system and that therefore the friction force is independent of the contact area. We have shown, however, that it is only the validity of the Bowden-Tabor law in Eq. (3) together with the linear load dependence of the contact area in Eq. (4) that leads to the Derjaguin-type friction-versus-load behavior seen in Fig. 3(c). As discussed before, this linear relationship between the load and the contact area cannot be taken for granted for any given contact situation.

Summarizing, we have shown here for the first time that the Bowden-Tabor term alone completely describes the kinetic friction in dry nanoscopic multiasperity systems even when wear is involved, and that the time evolution of the respective wear depth can be interpreted in terms of a combination of macroscopic Barwell laws. The observed linear load dependence of the friction force was found to be a result of the linear relationship between the contact area and the load.

This work was funded by the Austrian COMET-Program (Project K2, XTribology, No. 824187/849109) and carried out at the "Excellence Centre of Tribology." S. J. E. acknowledges the support of the Province of Niederösterreich (Project "SaPPS", WST3-T-8/0282014). G. F. acknowledges the support by the Austrian Science Fund (FWF): F4109 SFB ViCoM. Part of this work 
was supported by COST Action MP1303. The authors wish to thank J. Sequard-Base for his advice concerning experimental wear data and $\mathrm{M}$. Wolloch for reading the Letter and making many useful suggestions.

*eder@ac2t.at

[1] H. C. Meng and K. C. Ludema, Wear 181-183, 443 (1995).

[2] F. T. Barwell, Wear 1, 317 (1958).

[3] F. Preston, J. Soc. Glass Technol. 11, 214 (1927).

[4] R. Holm, Electrical Contacts (H. Gerbers, Stockholm, 1946).

[5] J. F. Archard, J. Appl. Phys., 24, 981 (1953).

[6] E. Rabinowicz, Friction and Wear of Materials (Wiley, New York, 1995).

[7] H.-J. Kim, S.-S. Yoo, and D.-E. Kim, Int. J. Precis. Eng. Man. 13, 1709 (2012).

[8] W. Maw, F. Stevens, S. C. Langford, and J. T. Dickinson, J. Appl. Phys. 92, 5103 (2002).

[9] B. Gotsmann and M. A. Lantz, Phys. Rev. Lett. 101, 125501 (2008).

[10] T. Jacobs and R. Carpick, Nat. Nanotechnol. 8, 108 (2013).

[11] B. Bhushan, Phil. Trans. R. Soc. A 366, 1499 (2008).

[12] G. Amontons, Mémoires de l'Académie Royale A 206 (1699).

[13] F. P. Bowden and D. Tabor, The Friction and Lubrication of Solids (Oxford University Press, Oxford, 1950).

[14] Y. Mo, K. T. Turner, and I. Szlufarska, Nature (London) 457, 1116 (2009).

[15] B. Luan and M. O. Robbins, Nature (London) 435, 929 (2005).

[16] S. J. Eder, D. Bianchi, U. Cihak-Bayr, A. Vernes, and G. Betz, Comput. Phys. Commun. 185, 2456 (2014).

[17] S. J. Eder, U. Cihak-Bayr, and A. Pauschitz, Wear, doi: 10.1016/j.wear.2015.03.006 (2015).

[18] S. J. Plimpton, J. Comput. Phys. 117, 1 (1995).

[19] M. I. Mendelev, S. Han, D. J. Srolovitz, G. J. Ackland, D. Y. Sun, and M. Asta, Philos. Mag. 83, 3977 (2003).

[20] A. Jabbarzadeh, P. Harrowell, and R. Tanner, J. Chem. Phys. 125, 034703 (2006)

[21] L. Ramin and A. Jabbarzadeh, J. Chem. Phys. 137, 174706 (2012).
[22] L. Pastewka, S. Moser, P. Gumbsch, and M. Moseler, Nat. Mater. 10, 34 (2011).

[23] L. J. Yang, Wear 259, 1453 (2005).

[24] R. Kumar, B. Prakash, and A. Sethuramiah, Wear 252, 445 (2002).

[25] H. Kloss and R. Wäsche, Wear 266, 476 (2009).

[26] X. Hu, C. J. Tourek, Z. Ye, S. Sundararajan, and A. Martini, Tribol. Lett. 53, 181 (2014).

[27] R. S. Montgomery, Wear 36, 275 (1976).

[28] G. Cameron and A. Palazotto, Wear 265, 1066 (2008).

[29] Y. Sesemann, C. Broeckmann, and A. Höfter, Wear 302, 1088 (2013).

[30] S. Eder, A. Vernes, G. Vorlaufer, and G. Betz, J. Phys. Condens. Matter 23, 175004 (2011).

[31] S. Eder, A. Vernes, and G. Betz, Comput. Phys. Commun. 185, 217 (2014).

[32] A. Vernes, S. Eder, G. Vorlaufer, and G. Betz, Faraday Discuss. 156, 173 (2012).

[33] S. J. Eder, A. Vernes, and G. Betz, Langmuir 29, 13760 (2013).

[34] L.-C. Zhang and H. Tanaka, JSME Int. J., Ser. A 42, 546 (1999).

[35] M. Wolloch, G. Feldbauer, P. Mohn, J. Redinger, and A. Vernes, Phys. Rev. B 91, 195436 (2015).

[36] A. Homola, J. Israelachvili, M. Gee, and P. McGuiggan, J. Tribol. 111, 675 (1989)

[37] R. W. Carpick, N. Agrait, D. Ogletree, and M. Salmeron, J. Vac. Sci. Technol. B 14, 1289 (1996).

[38] R. W. Carpick and M. Salmeron, Chem. Rev. 97, 1163 (1997).

[39] I. Szlufarska, M. Chandross, and R. W. Carpick, J. Phys. D 41, 123001 (2008).

[40] F. Bowden and D. Tabor, Proc. R. Soc. A 169, 391 (1939).

[41] L. Zhang and H. Tanaka, Tribol. Int. 31, 425 (1998).

[42] Y. Mo and I. Szlufarska, Phys. Rev. B, 81, 035405 (2010).

[43] J. Greenwood and J. Williamson, Proc. R. Soc. A 295, 300 (1966)

[44] M. Wolloch, G. Feldbauer, P. Mohn, J. Redinger, and A. Vernes, Phys. Rev. B 90, 195418 (2014). 